\title{
Upregulated 14-3-3ß aggravates restenosis by promoting cell migration following vascular injury in diabetic rats with elevated levels of free fatty acids
}

\author{
LISHUAI FENG ${ }^{1}$, CHAORAN DOU $^{2}$, JIANBO WANG $^{1}$, CHUNYU JIANG $^{1}, \mathrm{XU} \mathrm{MA}^{1}$ and JINGJING LIU ${ }^{1}$ \\ Departments of ${ }^{1}$ Interventional Radiology, Shanghai Jiao Tong University Affiliated Sixth People's Hospital, \\ ${ }^{2}$ Ultrasound in Medicine, Shanghai Institute of Ultrasound in Medicine, Shanghai Jiao Tong University \\ Affiliated Sixth People's Hospital, Shanghai 200233, P.R. China
}

Received April 28, 2017; Accepted May 9, 2018

DOI: $10.3892 / \mathrm{ijmm} .2018 .3671$

\begin{abstract}
Mono-unsaturated free fatty acids (FFAs) can serve as a predictive indicator of vascular restenosis following interventional therapy, particularly in individuals with high-fat diet-induced type 2 diabetes. However, the pathogenic mechanism remains to be fully elucidated. In the present study, the levels of tyrosine 3-monooxygenase/tryptophan 5 -monooxygenase activation protein $\beta$ (YWHAB; also known as $14-3-3 \beta$ ), in vascular smooth muscle cells (VSMCs) treated with different concentrations of oleic acid (OA) were examined by reverse transcription-quantitative polymerase chain reaction and western blot analyses. The migration of VSMCs was examined using wound-healing and Transwell migration assays. The protein distribution of B-cell lymphoma 2 (BCL-2)-associated death promoter (BAD) in VSMCs treated with $\mathrm{OA}$ was examined by immunofluorescence and western blot analyses. In in vivo experiments, the carotid artery morphology of rats in different groups was assessed at 14 days post-injury by non-invasive ultrasonographic imaging and confirmed by histological staining. The expression of YWHAB was upregulated by OA in a concentration-dependent manner in VSMCs. In the in vivo experiments, carotid stenosis was more serious among high-FFA diabetic rats. However, silencing of YWHAB significantly alleviated carotid neointimal hyperplasia among the diabetic rats with elevated FFA levels. In addition, YWHAB silencing alleviated the migration of OA-treated VSMCs and increased translocation of the BAD protein from the cytoplasm to the mitochondria. In conclusion, the results showed that FFA-induced upregulation
\end{abstract}

Correspondence to: Professor Jianbo Wang, Department of Interventional Radiology, Shanghai Jiao Tong University Affiliated Sixth People's Hospital, 600 Yishan Road, Shanghai 200233, P.R. China

E-mail: jeanbob_wang@163.com

Key words: restenosis, diabetes, free fatty acids, vascular smooth muscle cell, migration of YWHAB was involved in neointimal hyperplasia by enhancing the migration of VSMCs following carotid artery injury. The inhibition of YWHAB may serve as a novel potential pharmacological target for preventing vascular restenosis following interventional therapy in diabetic individuals with high FFA levels.

\section{Introduction}

Over 300,000,000 individuals have type-2 diabetes mellitus (T2DM) worldwide (1). T2DM magnifies the morbidity and mortality rates of cardiovascular diseases, including neuropathy, retinopathy and peripheral artery disease (2). Revascularization by endovascular techniques has become a preferred therapeutic option for these patients (3). However, vascular restenosis is considered to be the leading problem following interventional therapy (4). T2DM has also been reported to be a high risk factor for vascular restenosis following angioplasty (5). Excessive mono-unsaturated free fatty acids (FFAs), mostly induced by an imbalance between caloric intake and energy expenditure, contributes to insulin resistance and is highly associated with the prevalence of T2DM (6). Elevated levels of FFAs have also been considered the earliest and most sensitive risk factor for cardiovascular complications (7). FFA levels have been found to vary between 29.4 and 5.23\% among T2DM and non-T2DM cohorts (8). Although the association between T2DM and vascular restenosis is known, limited investigations of whether elevated FFA levels exacerbate this process, and the underlying mechanism, has been performed.

In general, abnormal growth of the intimal layer of blood vessels in response to injury is a key event in the development of vascular restenosis (9). During this process, excessive migration and proliferation of vascular smooth muscle cells (VSMCs) is an important step in neointimal formation and vascular remodeling in vivo $(10,11)$. Endovascular therapies induce endothelial denudation and VSMC exposure to inflammatory factors/cytokines, leading to the differentiation and migration of VSMCs (12). Owing to hyperglycemia in diabetes, the delayed repair of vascular endothelial cells (ECs) increases the risk of VSMC migration to the intima (13). In 
addition to high glucose toxicity, carotid artery stenosis is exacerbated in diabetic rats with higher levels of FFAs (14). Oleic acid (OA) is one of the most common FFAs in the blood of patients with diabetes, and a previous study demonstrated that excessive OA has an effect on vascular restenosis (15). To explain the association between high FFA levels and cardiovascular complications, OA is considered lipotoxic and is used to mimic high FFA content in in vitro experiments (16).

A thorough understanding of the mechanism underlying the association between FFA accumulation and VSMC migration in the development of vascular restenosis may provide clues for preventing or alleviating this pathological process. Our previous study demonstrated that tyrosine 3-monooxygenase/tryptophan 5-monooxygenase activation protein $\beta$ (YWHAB; also known as 14-3-3 $\beta$ ), was upregulated in ECs and was involved in neointimal hyperplasia in rats with diabetes induced by streptomycin alone (17). However, as YWHAB is non-specifically expressed in vivo, the role of YWHAB in VSMC migration and neointimal hyperplasia following injury remains to be elucidated. Therefore, the present study examined the role of YWHAB in vascular restenosis following carotid balloon injury in diabetic rats with high FFA levels. Furthermore, in vitro experiments were performed to understand the effect of YWHAB on FFA-induced VSMC migration. Finally, as YWHAB may exert a pro-survival effect by targeting the pro-apoptotic B-cell lymphoma 2 (BCL-2)-associated death promoter (BAD) protein, the effect of YWHAB on the subcellular localization of BAD was investigated.

\section{Materials and methods}

VSMC culture and infection. To determine an optimal OA concentration (Sigma-Aldrich; EMD Millipore, Billerica, MA, USA) for use in experiments in the present study, VSMCs (American Type Culture Collection, Manassas, VA, USA) were cultured in Dulbecco's modified Eagle's medium (DMEM; Gibco; Thermo Fisher Scientific, Inc., Waltham, MA, USA) containing 10\% fetal bovine serum (FBS; Gibco; Thermo Fisher Scientific, Inc.) and 0, 0.05, 0.1, 0.2, or $0.3 \mathrm{mM}$ OA. In another set of experiments, the VSMCs were cultured in DMEM supplemented with $10 \%$ and containing 5.6, 10, 15,25 , or $35 \mathrm{mM}$ glucose. Once the cells had reached at least $70 \%$ confluency, they were harvested and subjected to western blot analysis, reverse transcription-quantitative polymerase chain reaction (RT-qPCR) analysis, or a 3-(4,5-dimethylthiazol-2-yl)-2,5-diphenyltetrazolium bromide (MTT) assay. To mimic the diabetic internal environment in vitro, VSMCs treated with $0.2 \mathrm{mM}$ OA plus $25 \mathrm{mM}$ glucose were infected with a recombinant lentiviral vector expressing a short interfering RNA (siRNA) targeting YWHAB (sequence, GCUACAAAAAGUGCAUUUUGC; YWHAB-Lv) or with a control vector (Mock-Lv). YWHAB-Lv and Mock-Lv were provided by Professor Wheeler (Department of Physiology and Medicine, University of Toronto, Toronto, Ontario, Canada).

Animal protocols. The present study conformed to the Guide for the Care and Use of Laboratory Animals published by the US National Institutes of Health (NIH Publication no. 85-23, revised 1996). Male, 8-week-old male Sprague-Dawley (SD) rats (Shanghai SIPPR-Bk Lab Animal Co., Ltd., Shanghai, China) weighing $153.8 \pm 12.4 \mathrm{~g}$ were randomly separated into five groups ( $n=12$ per group), namely the normal, diabetic, high-fat diet, high-FFA diabetic, and YWHAB-silenced groups, based on the treatments they received. All rats were housed at a controlled temperature of $25^{\circ} \mathrm{C}$ under a $12 \mathrm{~h}$ light/dark cycle with ad libitum access to food and water. The rats in the normal and diabetic groups were fed a regular diet (60\% carbohydrates, $22 \%$ proteins, $10 \%$ fats), whereas the rats in the high-fat diet, high-FFA diabetic, and YWHAB-silenced groups were fed a high-fat diet $(50 \%$ carbohydrates, $13 \%$ proteins, $30 \%$ fats). After 6 weeks, the rats in the diabetic, high-FFA diabetic, and YWHAB-silenced groups were injected intraperitoneally with $30 \mathrm{mg} / \mathrm{kg}$ freshly prepared streptozotocin (STZ) solution (in $0.05 \mathrm{M}$ citrate buffer, $\mathrm{pH} 4.4$ ) to induce hyperglycemic symptoms, whereas the rats in the normal and high-fat diet groups received only the vehicle. At 3 days post-injection, the rats were fasted for $8 \mathrm{~h}$, and fasting blood glucose (FBG) levels were measured in blood samples collected from the tail veins. Irrespective of whether the FFA level was elevated, rats with an FBG $>11.1 \mathrm{mmol} / 1$ were considered diabetic. For viral infection, $100 \mu \mathrm{l}$ of either Mock-Lv or YWHAB-Lv $\left(10^{8} \mathrm{TU} / \mathrm{ml}\right)$ was then injected into the tail vein of rats in the high-FFA diabetic and YWHAB-silenced groups, respectively. At 2 weeks post-infection, six rats from each group were examined by non-invasive ultrasonography of the carotid arteries and then sacrificed for histomorphological analysis. The remaining rats were anesthetized with isoflurane. A 2-French balloon catheter (Edwards Lifesciences, Irvine, CA, USA) was inserted through the left external carotid artery into the common carotid artery and insufflated three times with 2 atm pressure. Following injury, the external carotid artery was rapidly ligated, and blood flow was resumed. The carotid arteries of all rats were examined by ultrasonography, and the rats were sacrificed at 14 days post-injury.

Ultrasonographic measurement of carotid arteries in vivo. Vascular ultrasonographic examination of the carotid arteries was performed in live rats using an ultrasonic diagnostic instrument (Mylab 90, Esaote, Genoa, Italy), which was operated by an experienced ultrasonographic technician. The rats were anesthetized with isoflurane and fixed on a mobile platform. Images of the carotid artery morphologies were captured longitudinally using $13 \mathrm{MHz}$ linear-array transducers on day 14 following carotid balloon injury.

Serum biochemical assays. The body weights and FBG levels were measured prior to the rats being sacrificed. Blood samples were collected from the tail vein. Total cholesterol (TC), FFAs, total triglyceride (TG), total low-density lipoprotein cholesterol (LDL-C), alanine transaminase (ALT), and aspartate transaminase (AST) from serum were assessed using enzyme-linked immunosorbent assay kits (Kehua Bio-engineering Co., Ltd., Shanghai, China).

Histomorphological and immunohistochemical staining. The injured segments of the left common carotid artery and the intact right carotid artery were dissected from the surrounding tissues, fixed in $10 \%$ formalin, embedded in paraffin, and stained with hematoxylin and eosin. The intimal and medial 
areas were measured using an image analyzer under a fluorescence microscope (Jenoptik, Jena, Germany) and calculated using the area-subtraction method. To confirm the role of VSMCs in the development of intimal hyperplasia, the injured segment of the left common carotid artery was immunostained with an antibody against the VSMC marker, $\alpha$-smooth muscle actin ( $\alpha$-SMA; cat. no. MAB1501R; diluted 1: 200; Sigma-Aldrich; EMD Millipore).

$R T-q P C R$ experiments. Total RNA was extracted using TRIzol (Invitrogen; Thermo Fisher Scientific, Inc.) and the chloroform/isopropanol method. cDNA was generated using the PrimeScript RT reagent kit (Takara Biotechnology Co., Ltd., Dalian, China), and qPCR was performed using SYBR Premix Ex Taq (Takara Biotechnology Co., Ltd.) with primers specific to YWHAB and glyceraldehyde-3-phosphate dehydrogenase (GAPDH). PCR reactions were performed in a total volume of $20 \mu \mathrm{l}$, including $2 \mu \mathrm{l}$ cDNA product, dNTPs (0.2 mM each), primers (20 pmol each) and 0.8 units Taq polymerase. PCR reactions were conducted under the following conditions: $95^{\circ} \mathrm{C}$ for $3 \mathrm{~min}$, followed by 40 cycles of $95^{\circ} \mathrm{C}$ for $30 \mathrm{sec}, 50-62^{\circ} \mathrm{C}$ for $30 \mathrm{sec}$ and $72^{\circ} \mathrm{C}$ for $1 \mathrm{~min}$. The qPCR data were normalized to the expression of GAPDH and presented as $2^{-\Delta \Delta \mathrm{Cq}}$ values (18). The primers used are as follows: YWHAB, forward 5'-CAAAGAGTACCGTGAGAA GATCGAG-3', and reverse 5'-CGGATGCAACTTCAGAAA GATACC-3'; GAPDH, forward 5'-GGCACAGTCAAGGCT GAGAATG-3' and reverse 5'-ATGGTGGTGAAGACGCCA GTA-3'.

Western blot analysis. Western blot analysis was performed to detect the expression of YWHAB and distribution of BAD in the VSMCs from the different groups. The lysates were isolated from each group using radio immunoprecipitation assay lysis buffer (Beyotime Institute of Biotechnology, Shanghai, China). The cytoplasmic and mitochondrial fractions were separated from crude cell lysates using the Mitochondria/Cytosol Fractionation kit (BioVision, Inc. Mountain View, CA, USA). The proteins (20 $\mu \mathrm{g} /$ lane) were separated by $10 \%$ sodium dodecyl sulfate polyacrylamide gel electrophoresis and transferred to polyvinylidene fluoride membranes (EMD Millipore). The membranes were blocked with 5\% skim milk in Tris-buffered saline Tween-20 (TBST) for $2 \mathrm{~h}$ and then incubated overnight at $4^{\circ} \mathrm{C}$ with the following diluted primary antibodies: Anti-GAPDH (cat. no. 2118; diluted 1:1,000; Cell Signaling Technology, Inc., Danvers, MA, USA); anti-YWHAB (cat. no. ab15260; diluted 1:1,000; Abcam, Cambridge, UK); Anti-BAD (cat. no. B0684; diluted 1:1,000; Sigma-Aldrich; EMD Millipore). The membranes were then washed three times with TBST, incubated for $2 \mathrm{~h}$ at $25^{\circ} \mathrm{C}$ with a horseradish peroxidase-conjugated secondary antibody (cat. no. A0545; diluted 1:5,000; Sigma-Aldrich; EMD Millipore), and washed with TBST. Gel images were obtained and analyzed using a gel imager (ChemiDoc XRS Image system; Bio-Rad Laboratories, Inc., Hercules, CA, USA) and Quantity One software (version 4.6.2; Bio-Rad Laboratories, Inc.). GAPDH was detected as a loading control.

Cell viability assays. MTT assays were performed to quantify VSMC proliferation. The VSMCs $\left(2.0 \times 10^{4}\right.$ cells/well $)$ were incubated in 96-well plates with growth medium containing $10 \%$ FBS. When the cells reached $70 \%$ confluency, they were starved for $12 \mathrm{~h}$ with $0.5 \%$ FBS and subsequently treated with as indicated in VSMC culture and infection section, for $36 \mathrm{~h}$. The VSMCs were incubated with the MTT reagent $(10 \mu 1$ per well) for $4 \mathrm{~h}$ and then with dimethyl sulfoxide $(150 \mu \mathrm{l}$ per well) for $10 \mathrm{~min}$, with shaking. Finally, the absorbance at $490 \mathrm{~nm}$ was measured using a microplate reader (Bio-Rad Laboratories, Inc.).

Wound-healing assays. The VSMCs were seeded in 6-well plates $(500,000$ cells/well) and grown for $24 \mathrm{~h}$ to reach at least $70 \%$ confluency. Following $24 \mathrm{~h}$ of serum deprivation, three parallel wounds of similar width $(<3 \mathrm{~mm})$ were created in each well using a sterile $200-\mu$ l pipette tip. The cells were incubated for $24 \mathrm{~h}$ to enable migration, and images of each well were captured under a fluorescence microscope (Jenoptik).

Transwell-migration assays. Following infection, the cells $\left(5 \times 10^{5}\right.$ cells $\left./ \mathrm{ml}\right)$ were resuspended in $200 \mu \mathrm{l}$ serum-free DMEM and then added to the upper chamber of a Transwell apparatus (Corning Incorporated, Corning, NY, USA). The lower chamber of the apparatus was filled with $500 \mu \mathrm{l}$ serum-free DMEM with Mock-Lv or YWHAB-Lv. After $16 \mathrm{~h}$, the migrated cells on the bottom surface of the chamber membrane were fixed with $4 \%$ paraformaldehyde and stained with $0.1 \%$ crystal violet at room temperature for $20 \mathrm{~min}$. The migrated cells were viewed with inverted light microscope (Nikon Corporation, Tokyo, Japan) and counted in five different fields of view.

Flow cytometry. Following infection, the cells were seeded overnight in 6-well plates $(500,000$ cells/well). Subsequently, they were collected by trypsinization, centrifuged $\left(4^{\circ} \mathrm{C}\right.$, $1,000 \times \mathrm{g}, 5 \mathrm{~min})$, resuspended and washed twice with $1 \mathrm{ml}$ precooled PBS, and then fixed overnight with $70 \%$ ethanol. The fixed cells were washed and resuspended in PBS, and consecutively treated with $20 \mathrm{ml}$ RNase A solution $\left(37^{\circ} \mathrm{C}\right.$, $30 \mathrm{~min})$ and $400 \mathrm{ml} \mathrm{PI}$ staining solution $\left(4^{\circ} \mathrm{C}, 60 \mathrm{~min}\right.$; Yeasen Biological Technology Co., Ltd., Shanghai, China), following which they were analyzed by flow cytometry.

Immunofluorescence. Following infection, mitochondria were labeled with $500 \mathrm{nM}$ MitoTracker ${ }^{\mathrm{TM}}$ (Invitrogen; Thermo Fisher Scientific, Inc.) for $30 \mathrm{~min}$ at $37^{\circ} \mathrm{C}$. Following three washes with PBS, the cells were fixed for 15 min with $4 \%$ paraformaldehyde and then permeabilized with $0.2 \%$ Triton $\mathrm{X}-100$ for $30 \mathrm{~min}$ at $20-25^{\circ} \mathrm{C}$. The cells were then washed with PBS and incubated with anti-YWHAB (1:200 dilution) and anti-BAD (1:200 dilution) primary antibodies at room temperature for $3 \mathrm{~h}$. The cells were washed twice in PBS and incubated with a FITC-conjugated secondary antibody (Santa Cruz Biotechnology, Inc., Santa Cruz, CA, USA) at room temperature for $1 \mathrm{~h}$. Coverslips were then mounted onto glass slides, and images were captured and analyzed using a fluorescence microscope.

Statistical analysis. All results were reproduced in at least six independent experiments and are presented as the mean \pm standard deviation. Statistical analysis was performed using the 8.0 SAS program (SAS Institute Inc., Cary, NC, 

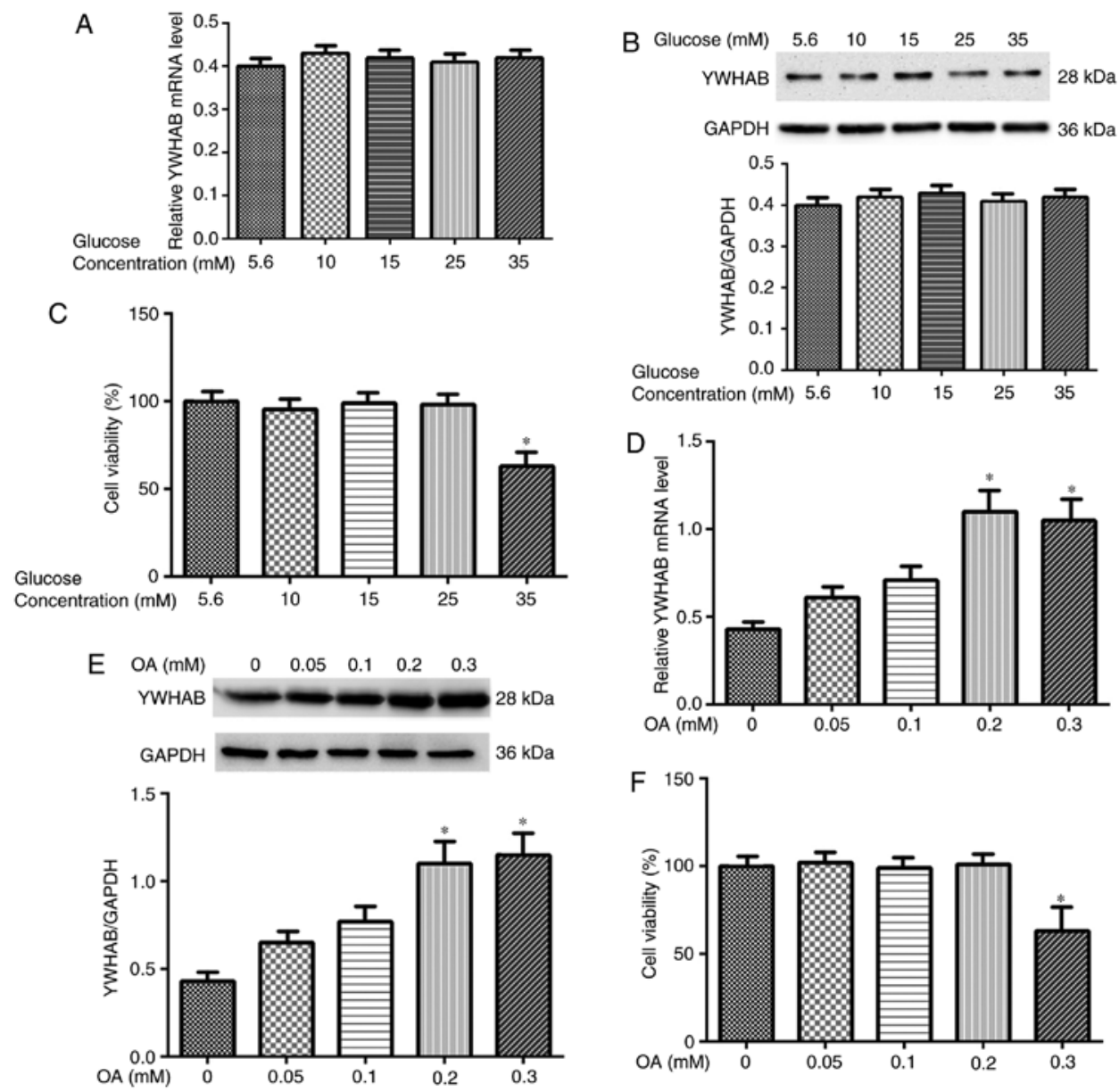

Figure 1. Effects of glucose and OA on expression of YWHAB and VSMC viability. (A) mRNA-expression levels of YWHAB in VSMCs treated with different glucose concentrations were detected by RT-qPCR analysis. (B) Representative western blot analysis and quantification of YWHAB and GAPDH levels treated with different glucose concentrations. (C) VSMC viability was detected by 3-(4,5-dimethyl-2-thiazolyl)-2,5-diphenyl-2-H-tetrazolium bromide assays. (D) RT-qPCR analysis was used to detect the mRNA expression levels of YWHAB following treatment with different OA concentrations. (E) Representative western blot analysis and quantification of YWHAB and GAPDH following treatment with different OA concentrations. (F) VSMC viability at different $\mathrm{OA}$ concentrations. ${ }^{~} \mathrm{P}<0.05$, vs. $0 \mathrm{mM}$ OA-treated group. Each experiment was repeated six times. VSMC, vascular smooth muscle cell; YWHAB, tyrosine 3-monooxygenase/tryptophan 5-monooxygenase activation protein $\beta$; OA, oleic acid; RT-qPCR, reverse transcription-quantitative polymerase chain reaction; GAPDH, glyceraldehyde 3-phosphate dehydrogenase.

USA). One-way analysis of variance with Student's t-test was applied to compare the differences between groups and estimate statistical significance. $\mathrm{P}<0.05$ was considered to indicate a statistically significant difference.

\section{Results}

Expression of $Y W H A B$ is upregulated with high $O A$ concentrations. Prior to performing in vivo experiments using rat VSMCs, in vitro experiments were performed in the present study using a rat cell line, with the rat VSMC line selected for this purpose. As OA is the most common and representative FFA found in plasma, it has been most frequently used to mimic the effects of FFAs in in vitro experiments (19). To exclude the effect of glucose on the expression of YWHAB, in vitro experiments were first performed to investigate whether different glucose concentrations affected the levels of YWHAB. As shown by the RT-qPCR and western blot analyses, no significant upregulation in the expression of YWHAB was observed in VSMCs with increasing glucose concentrations (Fig. 1A and B). The viability of the VSMCs decreased significantly when the cells were treated with $35 \mathrm{mM}$ glucose (Fig. 1C). However, the expression of YWHAB significantly increased in the VSMCs treated with OA (the most representative FFA) in a concentration-dependent manner (Fig. 1D and $\mathrm{E}$ ). There was no significant correlation between the levels of YWHAB and glucose concentration, but YWHAB was directly correlated with the OA concentration that the cells were exposed to. Notably, the viability of these cells did not alter at OA concentrations of up to $0.2 \mathrm{mM}$. However, when treated with $0.3 \mathrm{mM} \mathrm{OA}$, the viability of the VSMCs significantly decreased due to the toxicity of OA at that concentration (Fig. 1F). Therefore, to mimic the high-FFA diabetic environment in the in vitro experiments, $\mathrm{OA}$ at a concentration of $0.2 \mathrm{mM}$ plus $25 \mathrm{mM}$ glucose were used for the subsequent in vitro experiments.

$Y W H A B$ silencing alleviates vascular restenosis following carotid balloon injury in high-FFA diabetic rats. A high-fat diet combined with streptomycin injection can be used to establish a diabetic animal model accompanied by increased FFA levels (20). This method for developing model diabetic 
A

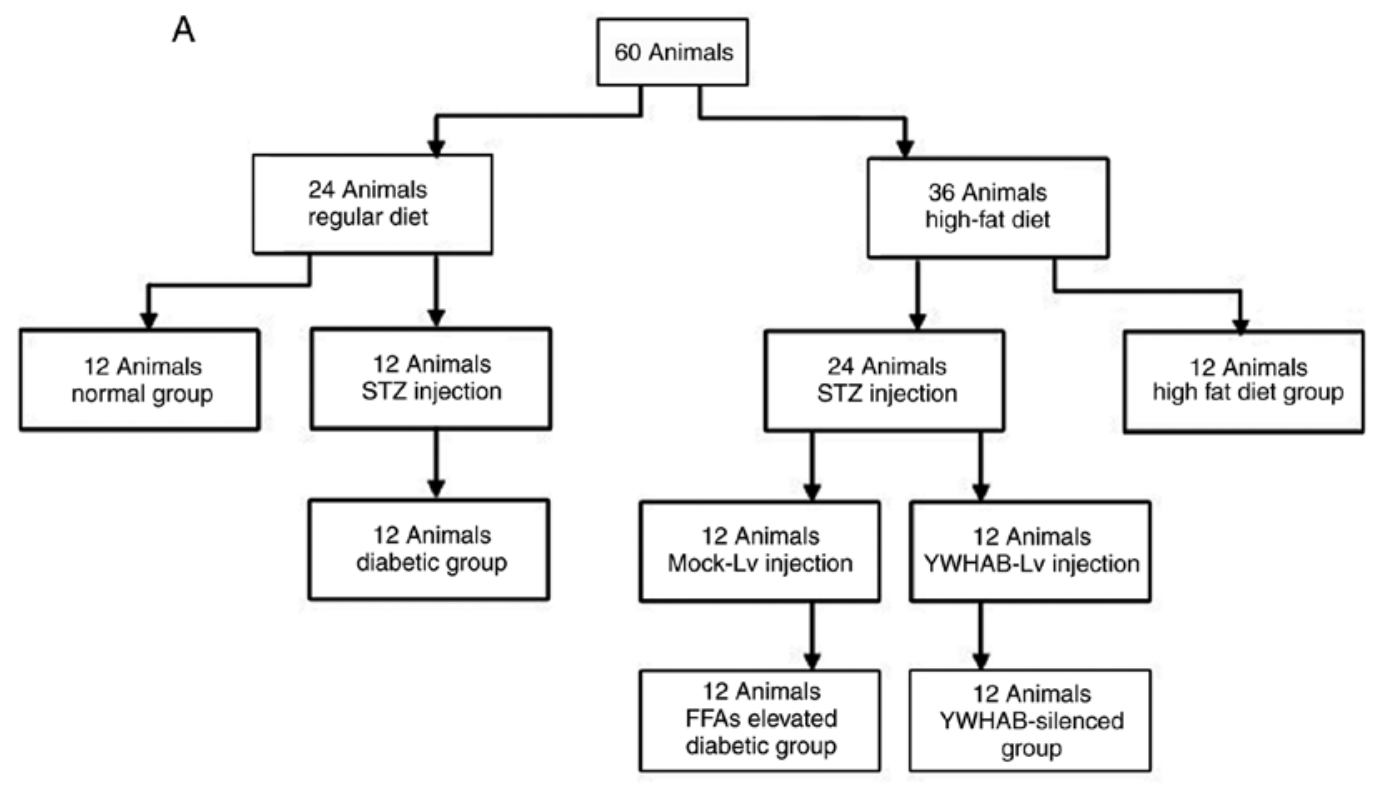

B

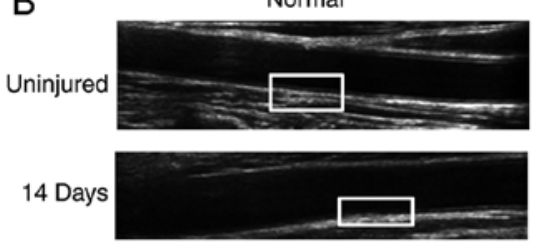

FFAs elevated diabetic
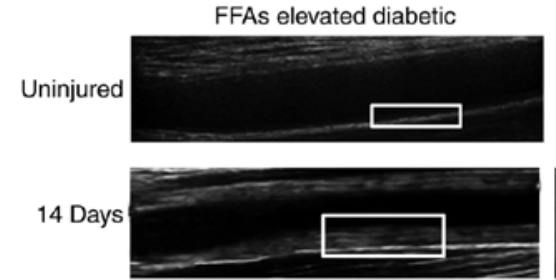

Diabetic

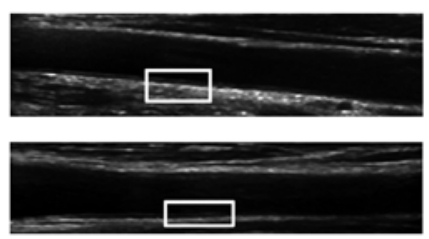

YWHAB-silenced

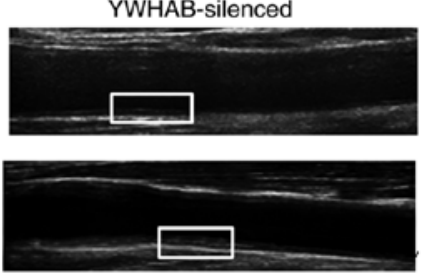

High fat diet
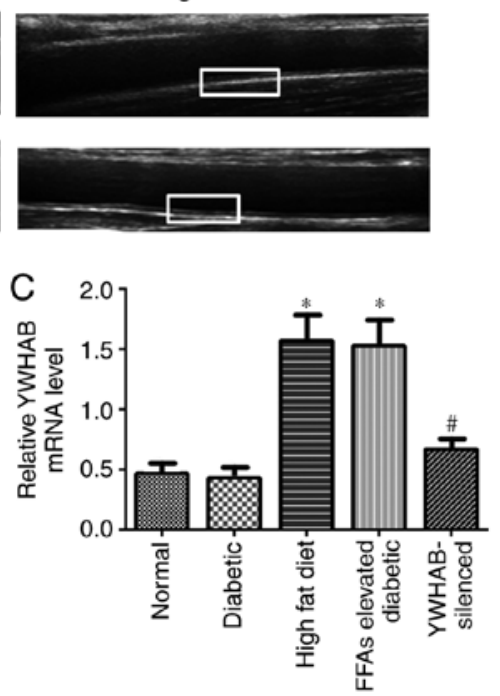

Figure 2. Ultrasonographic measurement of rat carotid arteries following vascular injury. (A) Flow chart of establishment of the animal model. (B) Representative images of rats' carotid arteries. The thicknesses of the wall layers are indicated with solid squares. (C) Expression of YWHAB was examined by reverse transcription-quantitative polymerase chain reaction analysis. ${ }^{*} \mathrm{P}<0.05$, vs. normal group; ${ }^{*} \mathrm{P}<0.05$, vs. high-FFA diabetic group. Each experiment was repeated six times. YWHAB, tyrosine 3-monooxygenase/tryptophan 5-monooxygenase activation protein $\beta$; FFAs, free fatty acids; STZ, streptozotocin.

animals has also been widely approved to observe changes in vascular restenosis following endovascular therapy $(21,22)$. This outcome can be explained by delayed EC healing owing to hyperglycemia. Without the protective effect of ECs, VSMCs are more inclined to migrate to the intima, leading to vascular restenosis following endovascular therapy (23). To exclude the individual effects of hyperglycemia and FFAs on neointimal hyperplasia following endovascular injury, five groups of rats were used, namely the normal, diabetic, high-fat diet, high-FFA diabetic, and YWHAB-silenced groups. A flow chart representing the establishment of the animal model is shown in Fig. 2A. The levels of FFA in the high-fat diet, high-FFA diabetic, and YWHAB-silenced groups were increased significantly compared with those in the normal and diabetic groups (Table I). Non-invasive ultrasonographic imaging technology was used to evaluate the carotid artery wall thickness prior to carotid artery injury and 14 days following injury. Consistent with the findings of a previous study (24), the level of carotid artery-stenosis was increased significantly in the high-FFA diabetic group, compared with that in the normal, diabetic, and high-fat diet groups (Fig. 2B). However, carotid artery stenosis was partly restored by YWHAB silencing (Fig. 2B). The levels of YWHAB in the intima were examined by RT-qPCR analysis. The results confirmed that YWHAB was upregulated in parallel with increased levels of FFAs (Fig. 2C).

$Y W H A B$ silencing prevents intimal hyperplasia following carotid balloon injury in high-FFA diabetic rats. To further confirm the above results, 14 days following the carotid balloon injury and lentivirus infection, the degree of neointimal hyperplasia was evaluated morphologically (Fig. 3A) and quantitatively. The intima and intima/media levels were 
A

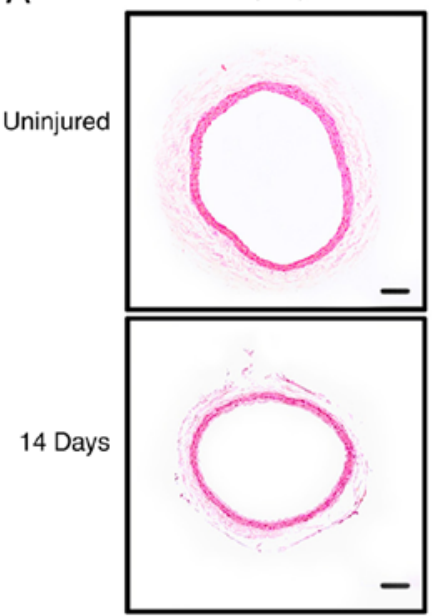

Diabetic

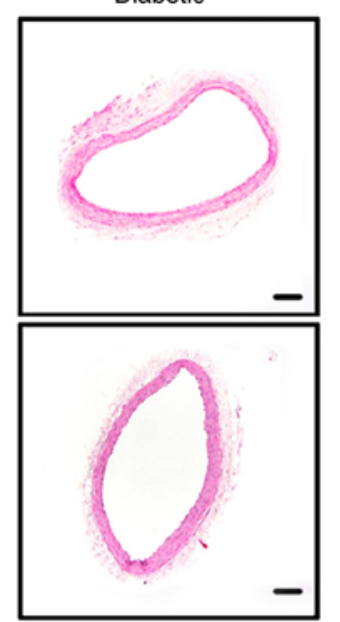

High fat diet

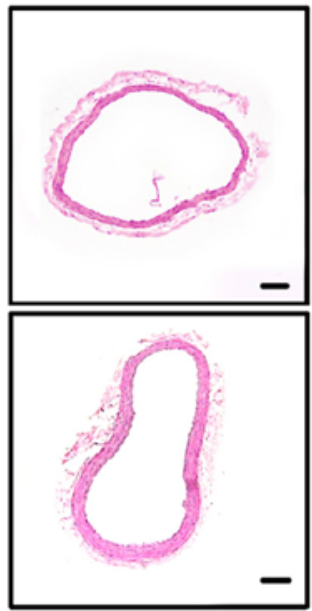

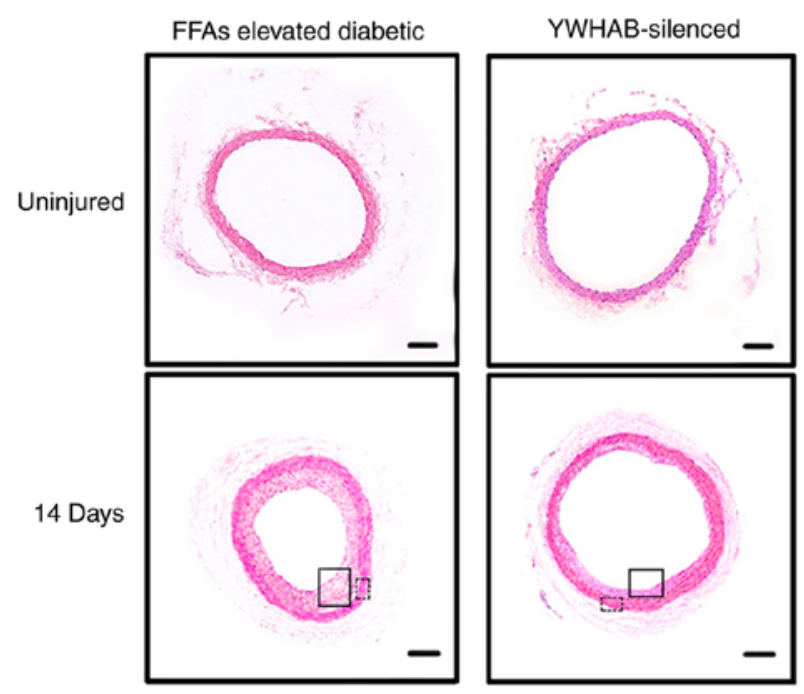
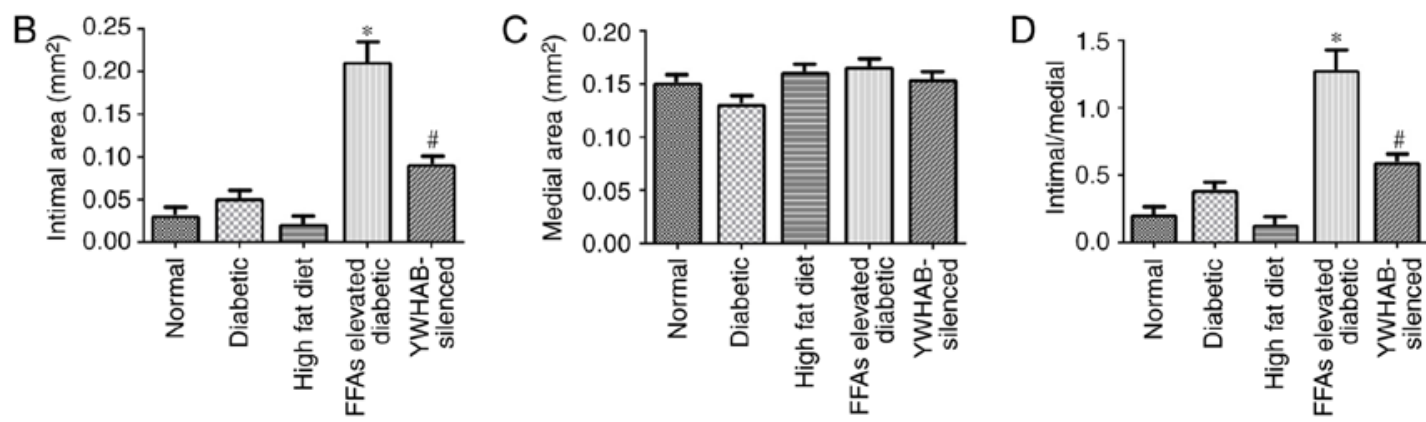

Figure 3. Effect of YWHAB on neointimal formation 14 days following vascular injury. (A) Representative cross-sections of hematoxylin/eosin staining in the experimental groups. (B) Graph indicating the intimal area. (C) Graph indicating the medial area. (D) Graph indicating the ratio of the intimal area to medial area. ${ }^{*} \mathrm{P}<0.05$, vs. normal group; ${ }^{\#} \mathrm{P}<0.05$, vs. high-FFA diabetic group. Each experiment was repeated six times. Scale bar $=25 \mu \mathrm{m}$. YWHAB, tyrosine 3 -monooxygenase/tryptophan 5-monooxygenase activation protein $\beta$; FFAs, free fatty acids.

increased significantly in the high-FFA diabetic group compared with those in the normal, diabetic, and high-fat diet groups, whereas no significant differences in the medial area were observed between any group (Fig. 3B-D). Specifically, YWHAB silencing significantly reduced the intimal area and the intima/media ratio (Fig. 3B and D) in the high-FFA diabetic rats. No significant differences in the medial area were observed in any group (Fig. 3C). These results were consistent with the ultrasonographic imaging findings. Immunohistochemistry staining against $\alpha$-SMA (a marker of
VSMCs) was also performed to confirm the role of VSMCs in the development of hyperlipidemia-induced intimal hyperplasia following carotid injury (Fig. 3E). In summary, the data convincingly confirmed that FFAs deteriorated vascular restenosis and YWHAB silencing attenuated high-FFA diabetic neointimal hyperplasia following carotid artery injury.

$Y W H A B$ silencing reduces the migration of OA-treated $V S M C s$. Subsequently, the present study examined the role of VSMCs in the development of hyperlipidemia-induced intimal 

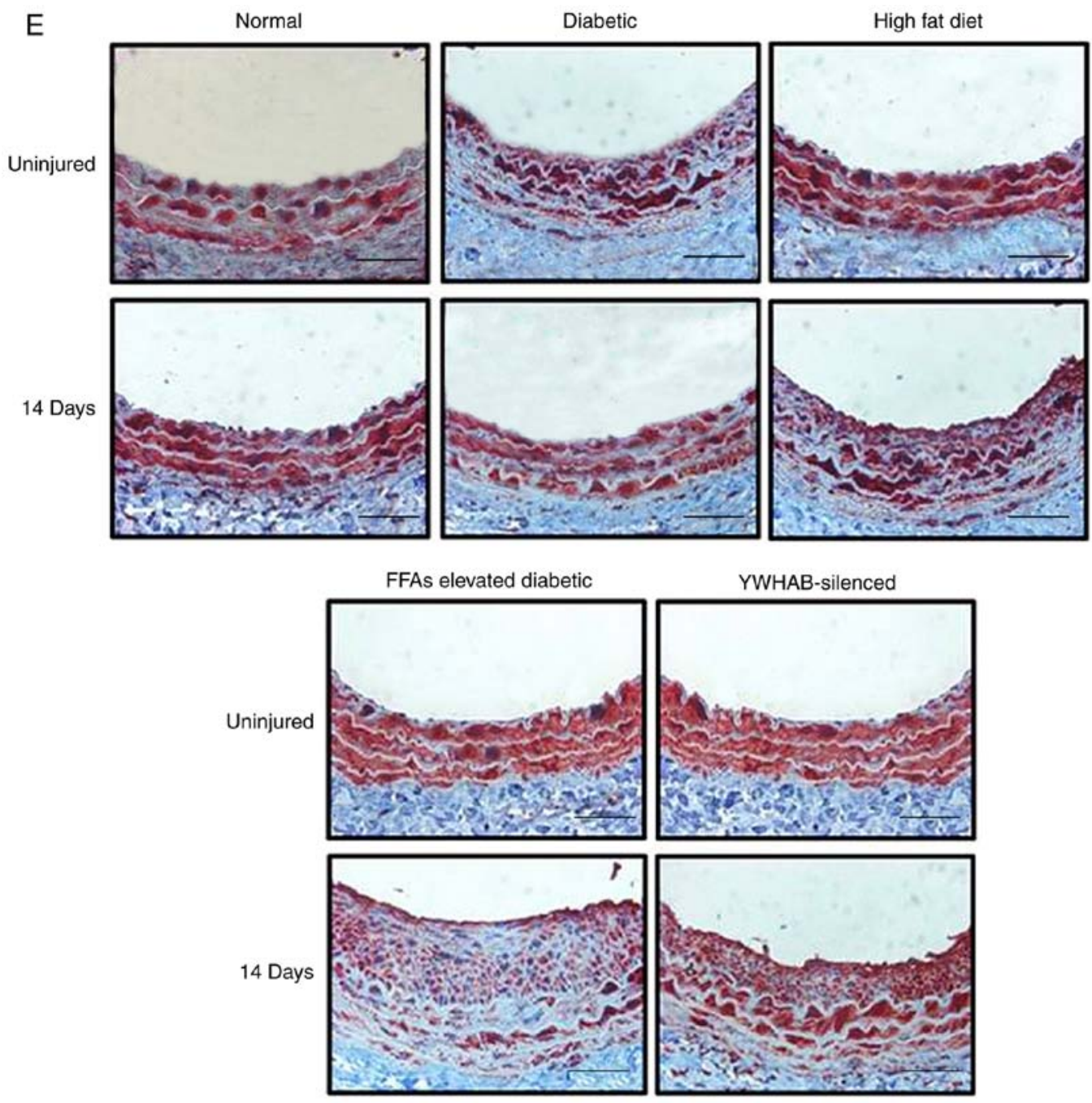

Figure 3. Continued. Effect of YWHAB on neointimal formation 14 days following vascular injury. (E) Tissue sections of rat carotid arteries 0 or 14 days following balloon injury were stained with $\alpha$-smooth muscle actin. Each experiment was repeated six times. Scale bar=25 $\mu \mathrm{m}$. YWHAB, tyrosine 3-monooxygenase/tryptophan 5-monooxygenase activation protein $\beta$; FFAs, free fatty acids.

Table I. Comparison of body weight and serum biochemical parameters between different groups of rats.

\begin{tabular}{lccccc}
\hline Parameter & Normal & Diabetic & High fat diet & High-FFAs diabetic & YWHAB-silenced \\
\hline Body weight $(\mathrm{g})$ & $177.53 \pm 3.83$ & $156.33 \pm 7.28$ & $247.28 \pm 1.82^{\mathrm{a}}$ & $241.26 \pm 1.32^{\mathrm{a}}$ & $238.22 \pm 2.74^{\mathrm{a}}$ \\
FBG (mmol/l) & $5.59 \pm 1.13$ & $13.78 \pm 1.53^{\mathrm{a}}$ & $5.68 \pm 1.75$ & $13.20 \pm 1.49^{\mathrm{a}}$ & $12.81 \pm 1.42^{\mathrm{a}}$ \\
AST (U/l) & $45.18 \pm 7.25$ & $57.79 \pm 17.22$ & $49.23 \pm 16.72$ & $56.72 \pm 17.32$ & $50.53 \pm 15.20$ \\
ALT (U/l) & $34.50 \pm 4.62$ & $35.65 \pm 7.32$ & $33.78 \pm 7.82$ & $36.72 \pm 10.15$ & $35.26 \pm 8.36$ \\
TG (mg/dl) & $34.10 \pm 6.90$ & $32.75 \pm 11.26$ & $39.78 \pm 12.56$ & $39.25 \pm 10.38$ & $39.48 \pm 11.26$ \\
TC (mg/dl) & $74.50 \pm 10.40$ & $70.46 \pm 17.84$ & $75.25 \pm 8.56$ & $72.36 \pm 9.25$ & $73.47 \pm 10.38$ \\
LDL-C (mg/dl) & $56.40 \pm 7.43$ & $50.26 \pm 8.76$ & $61.32 \pm 7.25$ & $60.78 \pm 10.25$ & $63.28 \pm 17.42$ \\
FFAs (mg/dl) & $17.73 \pm 3.71$ & $14.75 \pm 3.47$ & $93.29 \pm 5.82^{\mathrm{a}}$ & $92.68 \pm 1.69^{\mathrm{a}}$ & $81.90 \pm 3.51^{\mathrm{a}}$ \\
\hline
\end{tabular}

${ }^{\mathrm{a}} \mathrm{P}<0.05$ vs. normal group. YWHAB, tyrosine 3-monooxygenase/tryptophan 5-monooxygenase activation protein $\beta$; FBG, fasting blood glucose; TC, total cholesterol; FFAs, free fatty acids; TG, total triglyceride; LDL-C, total low-density lipoprotein cholesterol; ALT, alanine transaminase; AST, aspartate transaminase.

hyperplasia following injury. The VSMCs were incubated with $0.2 \mathrm{mM}$ OA and infected with the YWHAB-Lv or Mock-Lv adenovirus. The silencing efficiency of YWHAB-Lv was evaluated by western blot analysis (Fig. 4A); the expression of YWHAB in VSMCs treated without OA served as the control. The effect of YWHAB on VSMC migration was assessed 

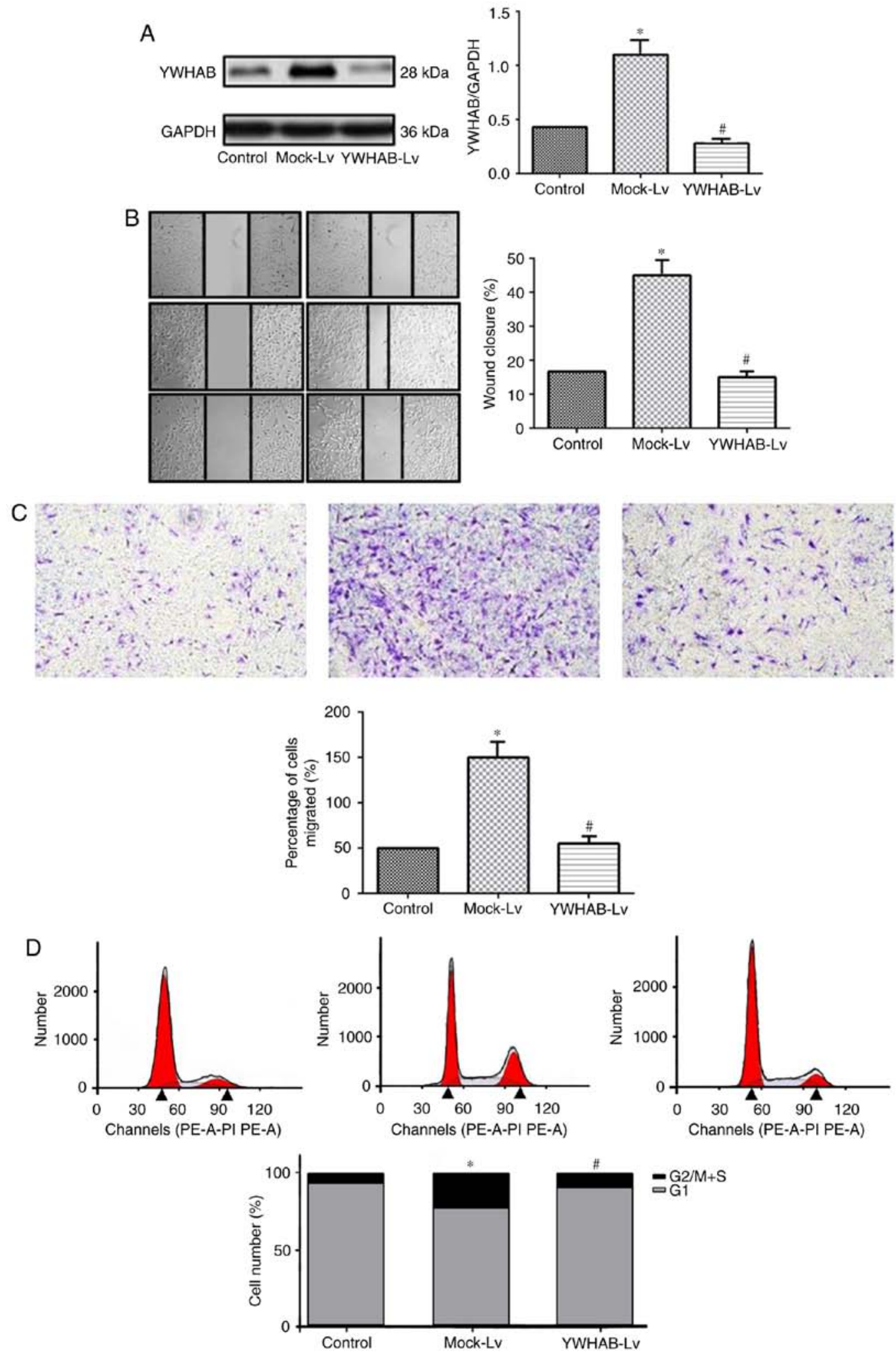

Figure 4. Effect of YWHAB silencing on the migration of VSMCs treated with OA. (A) Expression of YWHAB was examined by western blot analysis. (B) Cell migration was investigated using wound-healing assays. (C) Cell migration was investigated by performing Transwell-migration assays Magnification, x100. (D) Cell cycle progression was analyzed by flow cytometry. Expression of YWHAB in VSMCs not treated with OA was determined as a control. " $\mathrm{P}<0.05$, vs. control group; ${ }^{\#} \mathrm{P}<0.05$, vs. Mock-Lv group. Each experiment was repeated six times. Mock-Lv, mock-lentiviral vector; YWHAB-Lv, YWHAB-lentiviral vector; YWHAB, tyrosine 3-monooxygenase/tryptophan 5-monooxygenase activation protein $\beta$; FFAs, free fatty acids; OA, oleic acid; GAPDH, glyceraldehyde 3-phosphate dehydrogenase.

by performing wound-healing and Transwell-migration assays. The wound-healing assays revealed that YWHAB silencing significantly decreased cell migration, compared with that of the control (Mock-Lv) cells (Fig. 4B). Similar results were obtained in the Transwell-migration assays (Fig. 4C). Regarding the VSMCs, the excessive proliferation 

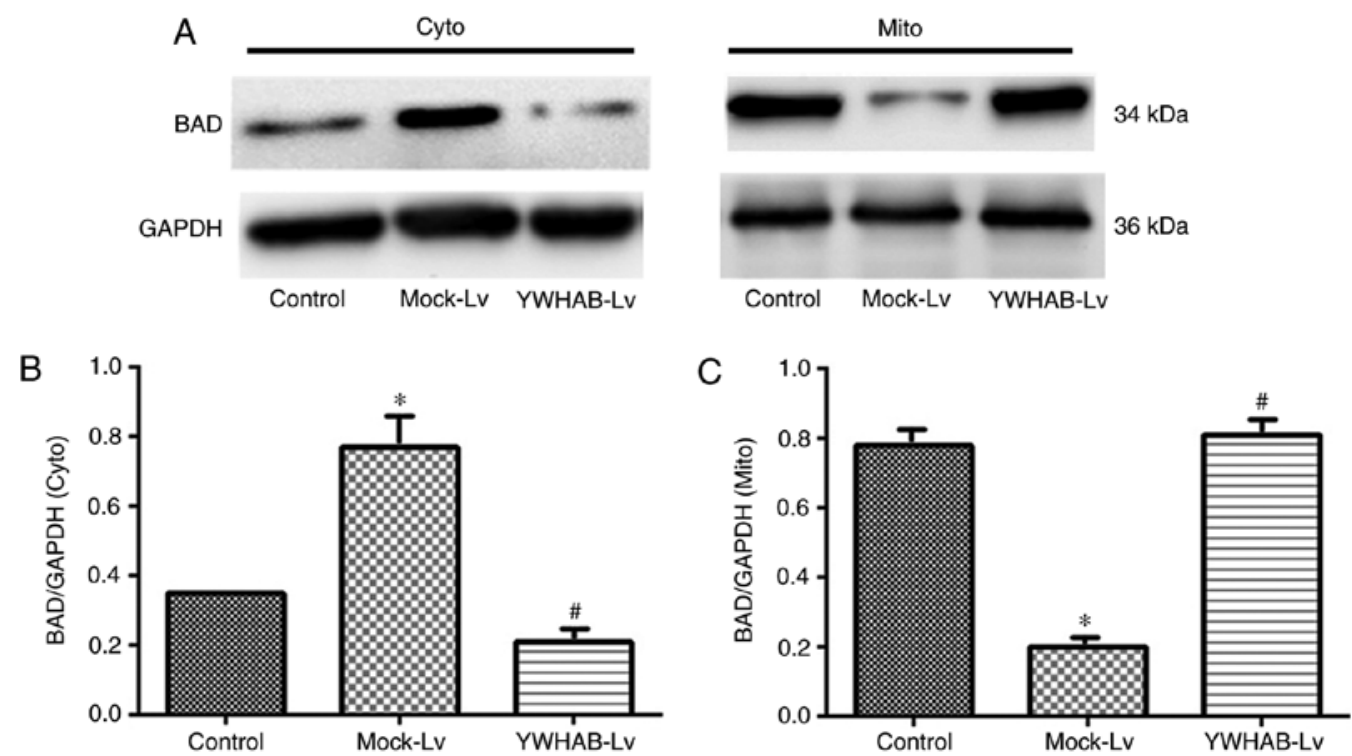

Figure 5. Effect of YWHAB silencing on expression of BAD in the Cyto and Mito fractions of VSMCs. (A) Expression of BAD was detected by western blot analysis. (B) Quantitative analysis of the expression of BAD in the Cyto fraction. (C) Quantitative analysis of the expression of BAD in the Mito fraction. The expression of BAD in cells treated with OA served as a control. ${ }^{*} \mathrm{P}<0.05$, vs. control group; ${ }^{*} \mathrm{P}<0.05$, vs. Mock-Lv group. Each experiment was repeated six times. Mock-Lv, mock-lentiviral vector; YWHAB-Lv, YWHAB-lentiviral vector; YWHAB, tyrosine 3-monooxygenase/tryptophan 5-monooxygenase activation protein $\beta$; BAD, B-cell lymphoma 2-associated death promoter; Cyto, cytoplasmic; Mito, mitochondrial; OA, oleic acid; GAPDH, glyceraldehyde 3-phosphate dehydrogenase.

and migration were closely associated with changes in cell cycle progression. Flow cytometry was performed to confirm the effect of YWHAB silencing on cell proliferation. The results of the flow cytometric analysis showed that YWHAB silencing significantly decreased cell cycle distribution in the $\mathrm{G} 2 / \mathrm{M}+\mathrm{S}$ phases, compared with that in the control (Mock-Lv) cells (Fig. 4D). These results indicated that YWHAB silencing reduced OA-induced VSMC proliferation and migration.

$Y W H A B$ silencing promotes the translocation of $B A D$ from the cytoplasm to the mitochondria. To investigate whether YWHAB affects BAD subcellular localization, the distribution of BAD was we examined in VSMCs treated with OA and in VSMCs in which the expression of YWHAB was silenced. In the control cells, BAD was predominantly localized in the mitochondria, where it regulated apoptosis (Fig. 5A-C). Treating the VSMCs with OA significantly decreased the BAD content in the mitochondria, compared with that in control cells with Mock-Lv. Immunofluorescent staining was also performed to confirm this result (Fig. 6A). These results indicated that YWHAB, the expression of which is induced by high-fat conditions, inhibited apoptosis by sequestering BAD in the cytoplasm, suggesting that YWHAB silencing may be involved in preventing excessive VSMC migration in a high-fat environment by causing the re-distribution of BAD to the mitochondria (Fig. 6B).

\section{Discussion}

In the present study, in vitro and in vivo experiments were performed which demonstrated that YWHAB had a significant role in neointimal hyperplasia by enhancing the migration of VSMCs following carotid artery injury. Patients with T2DM have a high risk for vascular restenosis following endovascular interventional therapy $(4,25,26)$. Imbalanced caloric intake is the major cause of T2DM, and previous data have indicated that excessive plasma FFA levels induced by a high fat intake are responsible for this pathological change (27). The upregulation of FFAs is a more sensitive indicator of excessive calorie intake than other blood lipid indices, including AST, ALT, and LDL-C levels. FFAs have been demonstrated to be pivotal in the native progression of atherosclerosis (28). The delayed recovery of ECs following endovascular therapy caused by a high glucose concentration increases the contact of inflammatory factors with VSMCs, which accelerates atherosclerosis. However, few studies have been performed to determine whether excessive plasma FFAs are also involved in accelerating the process. The present study confirmed this possibility by showing morphological differences between the carotid arteries of rats fed a normal diet and those fed a high-fat diet for 14 days following artery injury. The non-invasive and histological results showed significantly higher vascular restenosis in rats fed a high-fat diet and in diabetic rats with low-dose STZ-induced high FFA levels, compared with the other groups. High FFA levels are essential for the development of vascular restenosis. In general, VSMC migration to the intima is considered the most common cause of vascular restenosis following endovascular therapy (29). In agreement with this, the in vitro experiments performed in the present study showed that OA, the most common FFA, promoted the migration of VSMCs. Subsequently, the mechanism underlying this association was investigated and it was found that OA upregulated the expression of YWHAB in rat VSMCs in a concentration-dependent manner. Simultaneously, to exclude the effect of glucose concentration on the expression of YWHAB, the expression levels of YWHAB were analyzed in VSMCs treated with different glucose concentrations. The in vivo experiments confirmed that the high-fat diet increased 

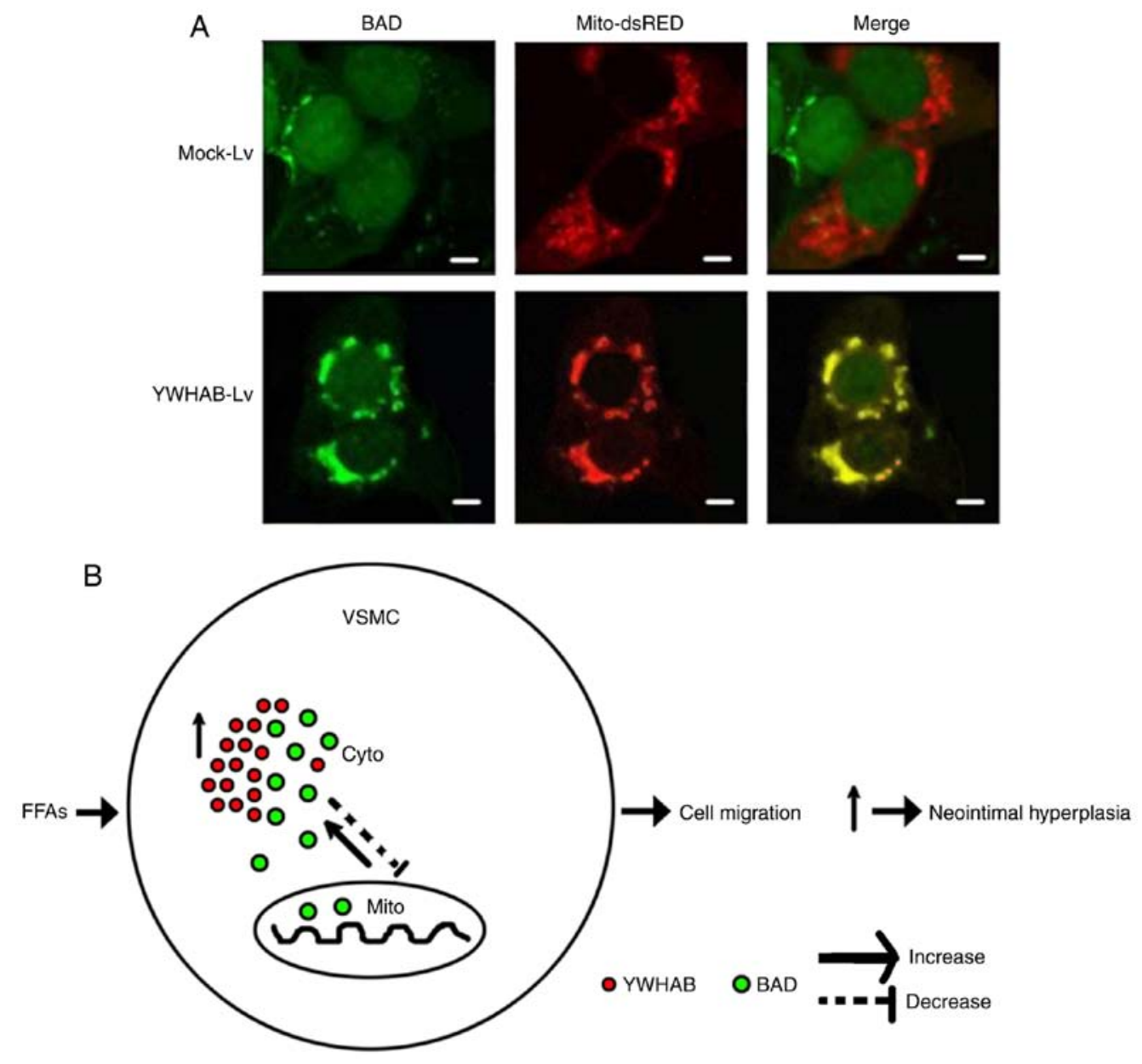

Figure 6. Effect of YWHAB silencing on the distribution of BAD. (A) Distributions of protein expression of BAD (green) and mitochondria (Mito-dsRED; red) were visualized by immunofluorescence staining. Scale bar=10 $\mu \mathrm{m}$. (B) Possible mechanism whereby YWHAB affects the sub-cellular localization of BAD in VSMCs treated with FFAs. Cyto, cytoplasm; Mito, mitochondria; YWHAB, tyrosine 3-monooxygenase/tryptophan 5-monooxygenase activation protein $\beta$; Mock-Lv, mock-lentiviral vector; YWHAB-Lv, YWHAB-lentiviral vector; BAD, B-cell lymphoma 2-associated death promoter; FFAs, free fatty acids; VSMC, vascular smooth muscle cell.

the level of YWHAB, whereas high glucose alone did not affect the expression of YWHAB in the intima.

YWHAB has previously been implicated in signal transduction, and promoting cell proliferation and the development of breast cancer $(30,31)$. Hartman and Hirsch (32) showed that the 14-3-3 protein family was involved in several diseases and biological processes through protein-protein interactions. Shimada et al (33) found that 14-3-3 proteins were beneficial for treating neurodegeneration by preventing neuron apoptosis by interacting with BAD or BCL-2-associated X protein. Data from our previous study indicated that YWHAB is a biomarker of intimal hyperplasia in type 1 diabetes mellitus SD rats following carotid artery injury (17). In the present study, using in vivo experiments, the association between the upregulation of YWHAB in high-FFA diabetic rats and vascular restenosis following carotid artery injury was examined. The results showed that YWHAB silencing alleviated the development of neointimal hyperplasia in the high-FFA diabetic group. To further elucidate the underlying mechanism, in vitro experiments were performed, which showed that YWHAB silencing decreased the migration of OA-treated VSMCs. Therefore, YWHAB is a biomarker for vascular restenosis following vascular injury. In high-FFA diabetic rats, the accumulation of fatty acids, eicosanoids, or oxidized phospholipids activates peroxisome proliferator-activated receptors (PPARs), which are involved in lipid metabolism. PPAR are expressed in three isoforms, namely PPAR $\alpha, \operatorname{PPAR} \gamma$ and PPAR $\delta$, which are encoded by different genes and form a subfamily of the nuclear receptor superfamily (34). The activation of PPARS in the VSMCs of diabetic patients with elevated levels of FFAs is beneficial to the cardiovascular system due to its effects on lipid metabolism, insulin sensitivity and glucose homeostasis $(35,36)$. Despite the necessity of PPAR $\delta$ activation during lipid metabolism, it was found that the activation of PPARS was induced by OA in hepatocellular carcinoma cells, which resulted in cellular metastasis by upregulating the expression of YWHAB and its interaction with BAD $(37,38)$.

$\mathrm{BAD}$ is a pro-apoptotic member of the Bcl-2 gene family, which is involved in initiating apoptosis; specifically, BAD is a member of the BH3-only subfamily (39). The BCL-2 and BCL-extra large (BCL-XL) proteins in the BCL-2 family have anti-apoptotic roles by inhibiting the release of cytochrome $c$ through the mitochondrial pore and activating the cytoplasmic caspase cascade (40). By contrast, BAD regulates the normal process of apoptosis by forming a heterodimer with BCL-2 and BCL-XL. Depending on the cell type, and the 
duration and magnitude of the stimulus, the mitochondrial subcellular localization of BAD can control a wide range of cell responses, including proliferation, migration, differentiation and cell death. The cytosolic binding of YWHAB with BAD and the generation of the BAD-YWHAB complex suppresses the mitochondrial translocation of BAD, which disrupts the effect of BAD on regulating apoptosis (38). In the present study, it was found that the level of BAD in the mitochondria of VSMCs exposed to OA was reduced, whereas YWHAB silencing reversed this effect and increased the levels of BAD in the mitochondria. These data may explain why VSMCs in the high-FFA diabetic rats or OA-treated cells showed an increased capacity for migration. Given the sophisticated mechanism of pathogenesis between lipid metabolism and diabetic complications, continued detailed investigations of the cellular and molecular mechanisms underlying the vascular complications in this disorder are required. The function of the YWHAB protein is closely associated with its post-translational modification and it is well known that YWHAB can regulate the functions of proteins by interacting with them in a phosphorylation-dependent manner. However, the detailed mechanism of how YWHAB regulates the expression of BCL-2 remains to be elucidated. Therefore, future investigations are required to explain the mechanism in detail.

In conclusion, the present study demonstrated that YWHAB was involved in neointimal hyperplasia in high-FFA diabetic rats following vascular injury. This effect was exerted through the induction of cell proliferation and retention of the pro-apoptotic factor BAD in the cytoplasm. These findings implicate YWHAB as a novel modulator of vascular restenosis, and highlight novel chemicals and targets for preventing vascular restenosis in diabetes characterized by high levels of FFAs.

\section{Acknowledgements}

Not applicable.

\section{Funding}

This study was financially supported by the Shanghai Shen Kang Hospital Development Center Suburb of Three Hospital Capacity Building projects (grant no. SHDC12014902), the Shanghai Key Subjects of Health System Project in Pudong New Area (grant no. PWZxq2014-07) and the Pudong New Area Science and Technology Development Fund (grant no. PKJ2016-Y52).

\section{Availability of data and materials}

The datasets used and/or analyzed during the current study are available from the corresponding author on reasonable request.

\section{Authors' contributions}

LF contributed to project development, manuscript writing, and data collection; CD contributed to ultrasound examination, data collection, and manuscript editing; JW contributed to conceptualization and funding acquisition; CJ contributed to data collection; XM contributed to methodology development; JL contributed to data analysis.

\section{Ethics approval and consent to participate}

This study was approved by the Ethics Committee of Shanghai Jiao Tong University Affiliated Sixth People's Hospital (Shanghai, China). The study conformed to the Guide for the Care and Use of Laboratory Animals published by the US National Institutes of Health (NIH Publication no. 85-23, revised 1996).

\section{Consent for publication}

Not applicable.

\section{Competing interests}

The authors declare that they have no competing interests.

\section{References}

1. Gong J, Fang K, Dong H, Wang D, Hu M and Lu F: Effect of fenugreek on hyperglycaemia and hyperlipidemia in diabetes and prediabetes: A meta-analysis. J Ethnopharmacol 194: 260-268, 2016.

2. Beckman JA, Creager MA and Libby P: Diabetes and atherosclerosis: Epidemiology, pathophysiology, and management. JAMA 287: 2570-2581, 2002.

3. Health Quality Ontario: Stenting for peripheral artery disease of the lower extremities: An evidence-based analysis. Ont Health Technol Assess Ser 10: 1-88, 2010.

4. Silva JA, Ramee SR, White CJ, Collins TJ, Jenkins JS, Nunez E, Zhang S and Jain SP: Primary stenting in acute myocardial infarction: Influence of diabetes mellitus in angiographic results and clinical outcome. Am Heart J 138: 446-455, 1999.

5. Breen DM and Giacca A: Effects of insulin on the vasculature. Curr Vasc Pharmacol 9: 321-332, 2011.

6. Gao A, Cayabyab FS, Chen X, Yang J, Wang L, Peng T and Lv Y: Implications of sortilin in lipid metabolism and lipid disorder diseases. DNA Cell Biol 36: 1050-1061, 2017.

7. Mathew M, Tay E and Cusi K: Elevated plasma free fatty acids increase cardiovascular risk by inducing plasma biomarkers of endothelial activation, myeloperoxidase and PAI-1 in healthy subjects. Cardiovasc Diabetol 9: 9, 2010.

8. Kuo SC,Lai SW, Hung HC, MuoCH, Hung SC, Liu LL, Chang CW, Hwu YJ, Chen SL and Sung FC: Association between comorbidities and dementia in diabetes mellitus patients: Population-based retrospective cohort study. J Diabetes Complications 29: 1071-1076, 2015.

9. Ashino T, Yamamoto $M$, Yoshida $T$ and Numazawa $S$ : Redox-sensitive transcription factor Nrf2 regulates vascular smooth muscle cell migration and neointimal hyperplasia. Arterioscler Thromb Vasc Biol 33: 760-768, 2013.

10. Nathan DM, Cleary PA, Backlund JY, Genuth SM, Lachin JM, Orchard TJ, Raskin P and Zinman B; Diabetes Control and Complications Trial/Epidemiology of Diabetes Interventions and Complications (DCCT/EDIC) Study Research Group: Intensive diabetes treatment and cardiovascular disease in patients with type 1 diabetes. N Engl J Med 353: 2643-2653, 2005.

11. Raines EW: PDGF and cardiovascular disease. Cytokine Growth Factor Rev 15: 237-254, 2004.

12. Bennett MR: In-stent stenosis: Pathology and implications for the development of drug eluting stents. Heart 89: 218-224, 2003.

13. Ma X, Jiang C, Li Y, Feng L, Liu J and Wang J: Inhibition effect of tacrolimus and platelet-derived growth factor-BB on restenosis after vascular intimal injury. Biomed Pharmacother 93: 180-189, 2017.

14. Wajima D, Nakagawa I, Takamura Y, Aketa S, Yonezawa T and Nakase H: Carotid artery stenosis is exacerbated in spontaneously obese model rats with diabetes. J Atheroscler Thromb 21: $1253-1259,2014$ 
15. Lin $\mathrm{MC}, \mathrm{Ou} \mathrm{TT}$, Chang $\mathrm{CH}$, Chan $\mathrm{KC}$ and Wang $\mathrm{CJ}$ : Protocatechuic acid inhibits oleic acid-induced vascular smooth muscle cell proliferation through activation of AMP-activated protein kinase and cell cycle arrest in $\mathrm{G}_{0} / \mathrm{G}_{1}$ phase. $\mathrm{J}$ Agric Food Chem 63: 235-241, 2015.

16. Plotz T, Krummel B, Laporte A, Pingitore A, Persaud SJ, Jörns A, Elsner M, Mehmeti I and Lenzen S: The monounsaturated fatty acid oleate is the major physiological toxic free fatty acid for human beta cells. Nutr Diabetes 7: 305, 2017.

17. Feng L, Ma X, Wang J and Tian Q: Up-regulation of 14-3-3beta plays a role in intimal hyperplasia following carotid artery injury in diabetic Sprague Dawley rats by promoting endothelial cell migration and proliferation. Biochem Biophys Res Commun 490: 1237-1243, 2017

18. Livak KJ and Schmittgen TD: Analysis of relative gene expression data using real-time quantitative PCR and the 2(-Delta Delta C(T)) method. Methods 25: 402-408, 2001.

19. Lager S, Gaccioli F, Ramirez VI, Jones HN, Jansson T and Powell TL: Oleic acid stimulates system A amino acid transport in primary human trophoblast cells mediated by toll-like receptor 4. J Lipid Res 54: 725-733, 2013.

20. Pedersen O, Kahn CR, Flier JS and Kahn BB: High fat feeding causes insulin resistance and a marked decrease in the expression of glucose transporters (Glut 4) in fat cells of rats. Endocrinology 129: 771-777, 1991.

21. Qi H, Jing Z, Xiaolin W, Changwu X, Xiaorong H, Jian Y, Jing C and Hong J: Histone demethylase JMJD2A inhibition attenuates neointimal hyperplasia in the carotid arteries of balloon-injured diabetic rats via transcriptional silencing: Inflammatory gene expression in vascular smooth muscle cells. Cell Physiol Biochem 37: 719-734, 2015.

22. Tsihlis ND, Rodriguez MP, Jiang Q, Schwartz A, Flynn ME, Vercammen JM and Kibbe MR: Nitric oxide differentially affects proteasome activator 28 after arterial injury in type 1 and type 2 diabetic rats. J Surg Res 202: 413-421, 2016.

23. Castilla DM, Liu ZJ, Tian R, Li Y, Livingstone AS and Velazquez OC: A novel autologous cell-based therapy to promote diabetic wound healing. Ann Surg 256: 560-572, 2012.

24. Wang H, Weihrauch D, Kersten JR, Toth JM, Passerini AG, Rajamani A, Schrepfer S and LaDisa JF Jr: Alagebrium inhibits neointimal hyperplasia and restores distributions of wall shear stress by reducing downstream vascular resistance in obese and diabetic rats. Am J Physiol Heart Circ Physiol 309: H1130-H1140, 2015.

25. Yang CP, Lin CC, Li CI, Liu CS, Lin WY, Hwang KL, Yang SY, Chen HJ and Li TC: Cardiovascular risk factors increase the risks of diabetic peripheral neuropathy in patients with type 2 diabetes mellitus: Medicine (Baltimore) 94: e1783, 2015.

26. Hong SJ, Kim MH, Cha KS, Park HS, Chae SC, Hur SH, Gwon HC, Bae JH and Lim DS: Comparison of three-year clinical outcomes between sirolimus-versus paclitaxel-eluting stents in diabetic patients: Prospective randomized multicenter trial. Catheter Cardiovasc Interv 76: 924-933, 2010.
27. Tishko VV, Sokolov AA, Belskih AN, Ivanov AM, Meshkova ME and Skorinova TS: Impact of double filtration plasmapheresis on adhesion molecules levels in patients with stable coronary heart disease after coronary stenting. Atheroscler Suppl 30: 92-98, 2017.

28. Plonka-Poltorak E, Zagrodzki P, Kryczyk-Koziol J, Westermarck T, Kaipainen P, Kaski M and Atroshi F: Does valproate therapy in epileptic patients contribute to changing atherosclerosis risk factors? The role of lipids and free fatty acids. Pharmacol Rep 68: 1339-1344, 2016.

29. Sun W, Huang Y, Yin T, Wang J, Du R, Qiu J, Zhang Y, Wang Y, Chen J and Wang G: Effects of elemene on inhibiting proliferation of vascular smooth muscle cells and promoting reendothelialization at the stent implantation site. Biomater Sci 5: 1144-1155, 2017.

30. Wilker E and Yaffe MB: 14-3-3 Proteins-a focus on cancer and human disease. J Mol Cell Cardiol 37: 633-642, 2004.

31. Zhang Y, Li M, Wei L, Zhu L, Hu S, Wu S, Ma S and Gao Y: Differential protein expression in perfusates from metastasized rat livers. Proteome Sci 11: 37, 2013

32. Hartman AM and Hirsch AKH: Molecular insight into specific 14-3-3 modulators: Inhibitors and stabilisers of protein-protein interactions of 14-3-3. Eur J Med Chem 136: 573-584, 2017.

33. Shimada T, Fournier AE and Yamagata K: Neuroprotective function of 14-3-3 proteins in neurodegeneration. Biomed Res Int 2013: 564534, 2013.

34. Gou Q, Gong X, Jin J, Shi J and Hou Y: Peroxisome proliferator-activated receptors (PPARs) are potential drug targets for cancer therapy. Oncotarget 8: 60704-60709, 2017.

35. Chen YC, Chu LY, Yang SF, Chen HL, Yet SF and Wu KK: Prostacyclin and PPAR $\alpha$ agonists control vascular smooth muscle cell apoptosis and phenotypic switch through distinct 14-3-3 isoforms. PLoS One 8: e69702, 2013.

36. Chu LY, Liou JY and Wu KK: Prostacyclin protects vascular integrity via PPAR/14-3-3 pathway. Prostaglandins Other Lipid Mediat 118-119: 19-27, 2015.

37. Bindesboll C, Berg O, Arntsen B, Nebb HI and Dalen KT: Fatty acids regulate perilipin 5 in muscle by activating PPAR $\delta$. J Lipid Res 54: 1949-1963, 2013.

38. Xu G, Wang J, Wu F, Wang N, Zhou W, Wang Q, Pan W, Ao G and Yang J: YAP and 14-3-3 $\gamma$ are involved in HS-OA-induced growth inhibition of hepatocellular carcinoma cells: A novel mechanism for hydrogen sulfide releasing oleanolic acid. Oncotarget 7: 52150-52165, 2016.

39. Adachi $\mathrm{M}$ and Imai K: The proapoptotic BH3-only protein BAD transduces cell death signals independently of its interaction with Bcl-2. Cell Death Differ 9: 1240-1247, 2002.

40. Tsuruta F, Masuyama N and Gotoh Y: The phosphatidylinositol 3-kinase (PI3K)-Akt pathway suppresses Bax translocation to mitochondria. J Biol Chem 277: 14040-14047, 2002. 